\title{
Associations of gene-wide SNPs in SNAI1 and TWIST1 with breast cancer and ovarian cancer susceptibility among Chinese Han women
}

\author{
YA-JUN WANG ${ }^{1}$, LI-YUAN ZHENG ${ }^{1}$, ZI-FAN WANG ${ }^{1}$, RUI SONG ${ }^{1}$, LI YANG ${ }^{1}$, YUE-HANG GENG ${ }^{1}$, \\ FANG MEI ${ }^{1}$, YUN-TAO XIE ${ }^{2}$, LAN CHEN ${ }^{3}$, DONG-GE LIU ${ }^{3}$, XIANG-HONG LI ${ }^{4}$, \\ YING-LI SUN $^{5}$, XIN-XIA TIAN $^{1}$ and WEI-GANG FANG ${ }^{1}$ \\ ${ }^{1}$ Department of Pathology, Key Laboratory of Carcinogenesis and Translational Research (Ministry of Education), \\ School of Basic Medical Sciences, Peking University Health Science Center, Beijing 100191; \\ ${ }^{2}$ Breast Center, Peking University School of Oncology, Beijing Cancer Hospital, Beijing 100142; \\ ${ }^{3}$ Department of Pathology, Beijing Hospital, Beijing 100005; ${ }^{4}$ Department of Pathology, \\ Peking University School of Oncology, Beijing Cancer Hospital, Beijing 100142; \\ ${ }^{5}$ Key Laboratory of Genomic and Precision Medicine, Chinese Academy of Sciences,
}

Beijing 100101, P.R. China

Received January 16, 2018; Accepted September 17, 2018

DOI: 10.3892/or.2018.6725

\begin{abstract}
Extensive evidence suggests that the genetic etiologies of breast cancer (BC) and ovarian cancer (OC) show a certain degree of similarity. This study aimed to find out whether the single nucleotide polymorphisms (SNPs) of genes SNAII and TWIST1 may affect BC and OC susceptibility. A total of 7 tagging-SNPs (tSNPs) were directly genotyped in 1,161 BC cases, 286 OC cases and 1,273 cancer-free controls among Chinese Han women. Twenty-eight variants in these 2 genes were genotyped by 'in silico' genotype imputation. Logistic regression (LR) revealed that tSNPs SNAII rs6125849, TWIST1 rs4721746 and TWIST1 rs4721745 were protective genetic variants for BC/OC. Allelic association tests of gene-wide SNPs demonstrated that the minor alleles of SNAII rs6125849, TWIST1 rs4721745 and TWIST1 rs11973396 were strongly associated with $\mathrm{BC} / \mathrm{OC}$ susceptibility. Multivariate LR presented that SNAII rs6125849, TWIST1 rs4721745, rs4721746 and rs11973396 affected BC/OC susceptibility independently, and women harboring all four protective genoytpes had the lowest risk. Multifactor dimensionality reduction analysis further showed that SNAII rs6125849 and
\end{abstract}

Correspondence to: Dr Xin-Xia Tian or Dr Wei-Gang Fang, Department of Pathology, Key Laboratory of Carcinogenesis and Translational Research (Ministry of Education), School of Basic Medical Sciences, Peking University Health Science Center, 38 Xueyuan Road, Beijing 100191, P.R. China

E-mail: tianxinxia@163.com

E-mail: wgfang@bjmu.edu.cn

Key words: SNAI1, TWIST1, breast cancer, ovarian cancer, single nucleotide polymorphism, Chinese Han women
TWIST1 rs4721745 had the strongest synergistic interaction. Functional annotation predicted that the minor alleles of SNAI1 rs6125849 and TWIST1 rs4721745 altered their binding affinities with transcription factors E2F6 and TCF11-MafG respectively. These results indicate that genetic variants in SNAII and TWIST1, most probably SNAI1 rs6125849 and TWIST1 rs4721745, may modulate BC and OC susceptibility.

\section{Introduction}

Breast cancer (BC) has become the most common malignancy among women worldwide (1), and ovarian cancer (OC) is the most lethal gynecological malignancy (2). Extensive evidence has shown that the etiologies of $\mathrm{BC}$ and $\mathrm{OC}$ are multifactorial involving hormonal, reproductive, environmental and genetic factors, and they may share certain genetic factors (3-5).

Genetic variants that influence the risk of $\mathrm{BC}$ and $\mathrm{OC}$ are divided into 3 categories: High-penetrant gene mutations, intermediate-penetrant gene mutations or variants, and low-penetrant variants (6). Approximately $20-30 \%$ of BC and $\mathrm{OC}$ cases exhibit familial aggregation, most of which are driven by low-frequency high-penetrant gene mutations, such as BRCA1, BRCA2, PTEN and TP53 (7,8). BRCA1 and $B R C A 2$ are involved in the homologous recombination pathway for double-strand DNA repair, maintaining genome stability (9). The PTEN gene encodes a phosphatase that negatively regulates the AKT/PKB signaling pathway, inducing cell cycle arrest, apoptosis and senescence (10). P53 is a tumor-suppressor gene involved in DNA repair, cell cycle arrest and apoptosis (11). Both family-based and population-based studies indicate that low-frequency genetic variants of DNA repair genes CHEK2, ATM, BRIP and PALB2 are associated with a moderate risk of breast cancer (12). In particular, the high-frequency low-penetrance genetic variants such as single 
nucleotide polymorphisms (SNPs) are more often associated with sporadic BC and OC (13).

Although the effect of a single SNP is generally small, several relevant SNPs may additively or synergistically contribute to increased breast cancer risk. On average, there is about one SNP for every 500 nucleotides in the human genome and each gene is covered by 52 SNPs (14), thus it is costly to genotype all these known SNPs in the target gene. Fortunately, based on linkage disequilibrium (LD), applying a much smaller subset of informative SNPs called haplotype-tagging SNPs (htSNPs) or tagging SNPs (tSNPs) can capture gene-wide common variations (15). Remarkably, by using these directly genotyped data of htSNP or tSNPs, many other variants that are not directly genotyped in laboratoriess can be genotyped on computer, an approach called genotype imputation (16). It predicts the individual genotypes at un-typed loci by comparing this individual to other individuals who shared a common haplotype or haplotypes, usually employing HapMap data as a reference (17). Therefore, htSNPs or tSNPs genotype analysis is a cost-effective strategy to pinpoint the polymorphisms of susceptibility genes in population association studies (18).

Snaill (encoded by gene SNAII) and Twist1 (encoded by gene $T W I S T 1$ ) are 2 key inducers of epithelial-mesenchymal transition (EMT). The SNAII gene is located on chr20:48,599,513-48,605,420 (human genome hg19 as reference) and its locus is 20q13.13. TWIST1 is located on chr7:19,155,091-19,157,295 (human genome hg19 as reference) and its locus is 7p21.1. Evidence indicates that Snail1 confers tumor cells with cancer stem cell-like traits, and promotes tumor recurrence, metastasis and drug resistance (19). It has been demonstrated that Snail1 plays a critical role in tumor growth and metastasis of breast and ovarian carcinoma by regulating MMP activity $(20,21)$. Twist1 is reported to be overexpressed in malignant and metastatic breast cancer (22) and ovarian cancer $(23,24)$. The positive expression of Twistl was found to be associated with poor progression-free survival and overall survival in epithelial ovarian carcinoma (24). Matsusaka et al analyzed associations between 7 functional SNPs in EMT-related genes (TWIST1, ZEB1, SNAII and E-cadherin) and outcomes in metastatic colorectal cancer patients. They found that female patients who carried the minor allele G of TWIST1 rs2285682 and TWIST1 rs2285681 had improved survival (25). However, little is known concerning the association between genetic polymorphisms of these 2 genes and BC/OC susceptibility.

In the present study, we genotyped 7 tagging SNPs, representatives of all SNPs within the SNAII and TWISTI genes, in 1,161 BC cases, 286 OC cases and 1,273 cancer-free female controls, and then performed a comprehensive correlation analysis between genotypes and cancer susceptibility. To fine-map the potential causal variants, genotype imputation was conducted to identify more SNPs that contribute to the susceptibility of the diseases in these two genes. Functional annotation predicted that SNAI1 rs6125849 and TWIST1 rs4721745 could be causal SNPs, which modulate BC and OC susceptibility.

\section{Materials and methods}

Study population and DNA isolation. This study included 1,161 BC cases and 978 cancer-free female controls, and 286 ovarian cancer cases and 295 cancer-free female controls.
All 1,447 cancer cases were recruited from Beijing Cancer Hospital,Peking University Third Hospital and Beijing Hospital between 1995 and 2010, and were pathologically diagnosed with breast invasive ductal carcinoma or ovarian serous carcinomas. The epidemiological information was extracted from their clinical records. The 978 cancer-free female controls and 295 cancer-free female controls, age-matched to BC and OC cases by 5-year age groups respectively, were selected from a community-based screening program for non-infectious diseases in Beijing. The epidemiological information of the controls was collected from the questionnaire. Age, height, weight, age at menarche and/or menopause, age at the first full-term pregnancy (FFTP) as well as family history of BC, $\mathrm{OC}$ or other cancers in first-degree relatives and other epidemiologic data were collected for all patients and controls.

For the 1,161 BC cases and all the 1,273 cancer-free female controls, genomic DNA was extracted from blood leukocytes as described by Grimberg et al (26). Briefly, $2 \mathrm{ml}$ of whole blood was mixed with $8 \mathrm{ml}$ of ice cold Triton lysis buffer $(0.32 \mathrm{M}$ sucrose, $10 \mathrm{mM}$ Tris- $\mathrm{HCl} \mathrm{pH} 7.6,5 \mathrm{mM}$ $\mathrm{MgCl}_{2}, 1 \%$ Triton X-100). After centrifugation, the nuclear pellet was resuspended in $500 \mu \mathrm{l}$ of proteinase $\mathrm{K}$ buffer $(1 \mathrm{mg} / \mathrm{ml}$ proteinase $\mathrm{K}, 10 \mathrm{mM}$ Tris- $\mathrm{HCl} \mathrm{pH} 8.0,10 \mathrm{mM} \mathrm{NaCl}$, $10 \mathrm{mM}$ EDTA) and incubated for $2 \mathrm{~h}$ at $65^{\circ} \mathrm{C}$. Genomic DNA was subsequently separated from proteins by saturated phenol, and then with phenol:chloroform:isoamyl alcohol (25:24:1) procedure. DNA in the supernatant fluid was precipitated by ethanol and dissolved in $400 \mu \mathrm{l}$ of TE buffer. DNA concentration was determined using NanoDrop 2000C (Thermo Fisher Scientific, Inc., Waltham, MA, USA) at $260 \mathrm{~nm}$.

For the 286 OC cases, genomic DNAs were extracted from archived formalin-fixed paraffin-embedded (FFPE) non-tumor tissues as described by Pikor et al (27). Briefly, for each case, 3 pieces of $10-\mu$ m-thick paraffin-embedded tissue sections were incubated in xylene to remove paraffin from the tissues, and then incubated in proteinase $\mathrm{K}$ buffer $(5 \mathrm{mg} / \mathrm{ml}$ proteinase $\mathrm{K}, 10 \mathrm{mM}$ Tris- $\mathrm{HCl} \mathrm{pH} 8.0,10 \mathrm{mM} \mathrm{NaCl}$ and $10 \mathrm{mM}$ EDTA) for 2 days until the tissues were fully dissolved. The DNAs were purified by phenol/chloroform DNA extraction method, and then precipitated by ethanol and dissolved in TE buffer. The DNA concentration was determined using NanoDrop 2000C at $260 \mathrm{~nm}$.

Selection of tagging-SNPS. To ensure good marker coverage of the entire genes and increase the analytic efficiency, tagging SNPs (tSNPs) were selected by Haploview v.4.2 software program based on the information of candidate genes in the HapMap database [HapMap Data Release \#27; Chinese Beijing population (CHB)] (www.hapmap.org). The tSNPs met the following requirements: A $0.8-\mathrm{r}^{2}$ threshold and a minor allele frequency $(\mathrm{MAF})>0.05$, and spanning from $2 \mathrm{~kb}$ upstream to $2 \mathrm{~kb}$ downstream for SNAII and TWIST1 genes, so that these tSNPs could capture all known common genetic variants within the entire gene. In the end, the following tSNPs were chosen, 3 tSNPs in SNAI1 (rs6125849, rs4647959, rs6020178) and 4 tSNPs in TWIST1 (rs2285682, rs2285681, rs4721746 and rs4721745).

Genotyping assay and quality control. Tagging-SNP genotyping was performed using TaqMan Assay ${ }^{\circledR}$ (Applied 
Biosystems, Foster City, CA, USA) using the ABI Step One ${ }^{\circledR}$ or ABI 7900HT ${ }^{\circledR}$ Real-Time PCR System (Applied Biosystems). Primers and probes (FAM- and VIC-labeled) were supplied directly by Applied Biosystems as Assays-by-Design and Assays-on-Demand products. Briefly, all assays were carried out in 48-well or 384-well plates with positive and negative controls in each genotyping plate. Plates were sealed and heated at $95^{\circ} \mathrm{C}$ for $5 \mathrm{~min}$, and then were subjected to $45-50$ cycles of $92^{\circ} \mathrm{C}$ for $15 \mathrm{sec}$ and $60^{\circ} \mathrm{C}$ for $1 \mathrm{~min}$. As for quality control, we repeated the genotyping on $3 \%$ of the samples. The concordance rate between duplicates was higher than $99 \%$.

Univariate and multivariate analyses. The distribution of categorical variables and continuous variables between cases and control groups was compared by Pearson's $\chi^{2}$ test and Student's t-test, respectively. For each SNP, Hardy-Weinberg equilibrium was evaluated using a one-degree of freedom goodness-of-fit $\chi^{2}$ test to compare the observed with the expected frequency of genotypes among the female controls. LD plots of the D' values for these SNPs were produced using the Haploview program (28). The most probable haplotypes of each participant were estimated using the expectation-maximization (EM) algorithm. For SNP analysis, we tested 3 different genetic models, namely dominant model, recessive model and codominant model, to identify the best-fitting one with the smallest P-value (29). Two-sided chi-square test was also used to investigate the differences in the distributions of genotypes between cases and controls. To analyze the associations of an individual SNP with BC and OC risk, univariate and multivariate logistic regression models were used to estimate the odds ratios (ORs) and 95\% confidence intervals (CIs). The above statistical analyses were performed by Statistic Analysis System software (SAS v9.1; SAS Institute, Cary, NC, USA).

False-positive report probability and statistical power. We calculated the false-positive report probability (FPRP) values for the statistically significant associations. We set 0.2 as the FPRP threshold and adopted a prior probability of 0.1 to detect OR of 1.50/0.67 (risk/protective effects) as described previously $(30,31)$. The association that reached the FPRP threshold of $<0.2$ was considered noteworthy.

Genotype imputation. Genotype imputation is used to predict the genotypes at the variants that are not directly assayed in the study sample (32). In this study, we used MACH software (http://csg.sph.umich.edu/abecasis/MACH/tour/imputation.html) to complete genotype imputation. To obtain more reliable results, we constructed reference haplotypes from the $\mathrm{CHB}$ population according to 1000 Genomes datasets. What's more, the module of imputation helper in GenGen software tool (http://gengen.openbioinformatics.org) was used to facilitate the analysis of high-throughput genomics datasets. Imputed variants that had an R-square measure $<0.3$ were excluded. Well-imputed variants were then genotyped and used to analyze the associations between the variants and $\mathrm{BC} / \mathrm{OC}$ susceptibility.

Multifactor dimensionality reduction (MDR) analysis. MDR analysis was conducted to identify higher-order interactions that were associated with cancer risk using MDR software (v.2.0 beta 8.4) (http://sourceforge.net/projects/mdr/). MDR analysis collapsed multi-dimensional data into a single independent dimensional variable with two levels (high and low risk) using the ratio of the number of cases to the number of controls, and thereby reduced multiple dimensional data into one dimension and permitted detection of interactions in relatively small sample sizes (33). MDR was also performed in cross-validation and permutation test to classify and predict disease status. The best candidate interaction model was selected to maximize testing accuracy and cross-validation consistency (CVC) (34).

Functional annotation tools. To explore the functional relevance of the selected SNPs, we annotated each variant using publicly available bioinformatics data and existing functional annotation software. HaploReg (http://www.broadinstitute. org/mammals/haploreg/haploreg.php) and RegulomeDB (http://regulome.stanford.edu/) provide annotation for variations, including the ENCODE project data, DNase hypersensitivity data, histone modification data, transcription factor ChIP-seq data, and eQTL datasets. Furthermore, we analyzed the ENCODE project (https://www.encodeproject.org/) data very closely in the breast cancer cell lines (HMEC and MCF-7) by using the UCSC Genome Browser (http://genome.ucsc.edu), which may indicate similar roles in ovarian cancer.

Ethics statement. This study was approved by the Peking University IRB (reference no. IRB00001052-11029). We obtained written informed consents from all BC cases and control women. Because we could not obtain the contact information of ovarian cancer patients treated before 2011, PKU IRB approved our application to waive informed consent for the archived samples collected before April 2011. This study only used these samples. We used all the data/samples anonymously.

\section{Results}

Characteristics of the study population. This study included 1,161 BC cases and 978 cancer-free female controls, 286 ovarian cancer cases and 295 cancer-free female controls. Since the etiology of $\mathrm{BC}$ and $\mathrm{OC}$ has similarity, we merged the $\mathrm{BC}$ cases and $\mathrm{OC}$ cases (designated as $\mathrm{BC} / \mathrm{OC}$ ) as well as control groups to increase the sample size. As shown in Table I, the 1,447 BC/OC patients and 1273 controls appeared to be adequately matched in age $(\mathrm{P}=0.1673)$. The patients had a much younger age at menarche $(\mathrm{P}<0.0001)$ and an elder age at first full-term pregnancy $(\mathrm{P}<0.0001)$ compared with the controls. In addition, a higher proportion of women were in pre-menopause status in the case group than that noted in the controls. For other characteristics such as body mass index (BMI), age at menopause and family history of cancer in first-degree relatives, no statistically differences were found $(\mathrm{P}>0.05)$. These variables were further used in multivariate logistic regression to adjust for any possible confounding effect on $\mathrm{BC} / \mathrm{OC}$ risk.

Similarly, the selected characteristics were separately analyzed in the BC and OC cases. The mean ages of the $1,161 \mathrm{BC}$ cases at diagnosis and the 978 cancer-free female controls were $49.28 \pm 10.53$ and $48.61 \pm 8.25$, respectively. They 
Table I. Characteristics of the BC/OC patients and cancer-free controls

\begin{tabular}{|c|c|c|c|}
\hline Variable & Case, $n=1,447$ & Control, $\mathrm{n}=1,273$ & P-value \\
\hline Age, years $($ mean $\pm S D)$ & $50.33 \pm 11.00$ & $49.79 \pm 9.05$ & 0.1673 \\
\hline Age, n (\%), years & & & 0.0742 \\
\hline$<50$ & $753(52.04)$ & $706(55.46)$ & \\
\hline$\geq 50$ & $694(47.96)$ & $567(44.54)$ & \\
\hline $\begin{array}{l}\text { Body mass index }(\mathrm{BMI}), \\
(\text { mean } \pm \mathrm{SD})\end{array}$ & $24.48 \pm 3.32$ & $24.65 \pm 3.49$ & 0.1915 \\
\hline $\begin{array}{l}\text { Age at menarche, years } \\
(\text { mean } \pm \mathrm{SD})\end{array}$ & $14.58 \pm 1.88$ & $15.12 \pm 1.91$ & $<0.0001$ \\
\hline $\begin{array}{l}\text { Age at menopause, years } \\
(\text { mean } \pm \text { SD) }\end{array}$ & $49.28 \pm 4.20$ & $49.22 \pm 3.80$ & 0.8150 \\
\hline $\begin{array}{l}\text { Age of first full-term pregnancy } \\
(\text { FFTP), years }(\text { mean } \pm \text { SD) }\end{array}$ & $26.03 \pm 2.91$ & $25.40 \pm 2.79$ & $<0.0001$ \\
\hline Menopause status, n (\%) & & & 0.0001 \\
\hline Premenopause & 827 (57.75) & $633(50.36)$ & \\
\hline Postmenopause & $605(42.25)$ & $624(49.64)$ & \\
\hline $\begin{array}{l}\text { Family history of cancer in } \\
\text { first-degree relatives, } \mathrm{n}(\%)\end{array}$ & & & 0.1374 \\
\hline Yes & $310(21.47)$ & 244 (19.17) & \\
\hline No & $1134(78.53)$ & $1029(80.83)$ & \\
\hline
\end{tabular}

Bold numbers indicate a statistical significance at the 0.05 level. BC, breast cancer; OC, ovarian cancer.

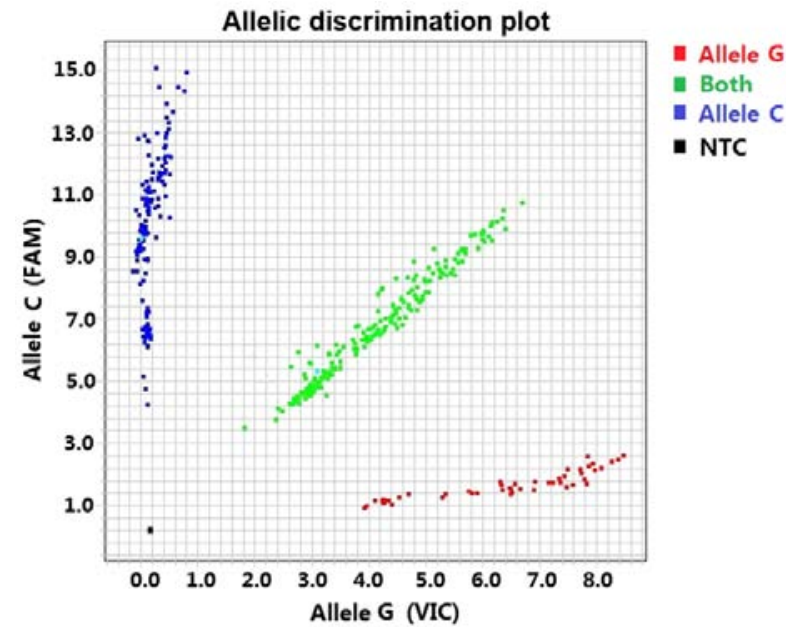

Figure 1. Representative genotyping results of TWIST1 rs4721745 (C>G) by using ABI HT7900 and a 384-well plate. Probe detecting C allele was labeled with FAM, and probe detecting G allele was labeled with VIC. Blue squares denote DNA samples with homozygous CC genotype, red squares denote DNA samples with homozygous GG genotype, and green square denoted DNA samples with heterozygous CG genotype. NTC, negative control.

were adequately matched in age $(\mathrm{P}=0.1037)$. $\mathrm{BC}$ patients had a much younger age at menarche $(\mathrm{P}<0.0001)$ and an elder age at primiparity $(\mathrm{P}<0.0001)$ compared with controls.

The mean ages of the $286 \mathrm{BC}$ cases at diagnosis and the 295 cancer-free female controls were $54.57 \pm 11.85$ and $53.71 \pm 10.40$, respectively. They were adequately matched in age $(\mathrm{P}=0.3517)$. The OC cases had a lower BMI $(\mathrm{P}=0.0309)$, younger age at menarche $(\mathrm{P}=0.0020)$ and higher proportion of post-menopausal individuals $(\mathrm{P}<0.0001)$ than controls.

Association of individual tSNPs in SNAII and TWIST1 with $B C$ and $O C$ risk. SNPs were genotyped by TaqMan Assay ${ }^{\circledR}$ (Applied Biosystems) using the Real-Time PCR System ABI Step One (48-well plate) or ABI 7900HT (384-well plates) (Fig. 1).

The 7 tSNPs in SNAII and TWISTI were all in agreement with Hardy-Weinberg equilibrium (HWE) in the control population $(\mathrm{P}>0.05)$.

As shown in Table II, there were significant differences in genotype distribution between BC/OC cases and controls in polymorphisms SNAII rs6125849 (G>A) and TWIST1 rs4721745 $(\mathrm{C}>\mathrm{G})$ by $\chi^{2}$ test $(\mathrm{P}=0.0128$ and 0.0007 respectively). Multivariate logistic regression showed that SNAII rs6125849, TWIST1 rs4721746 and TWIST1 rs4721745 were all associated with decreased $\mathrm{BC} / \mathrm{OC}$ risk in the dominant model after adjusting for body mass index (BMI), age at menarche, age of first full-term pregnancy and menopause status (adjusted odds ratio, $\mathrm{aOR}=0.77, \mathrm{P}=0.0087 ; \mathrm{aOR}=0.83, \mathrm{P}=0.0434$; $\mathrm{aOR}=0.73, \mathrm{P}=0.0002$, respectively). Moreover, the cancer risk was further decreased with the increase in minor allele dose for the 3 tagging SNPs $\left(\mathrm{P}_{\text {trend }}=0.0038,0.0492\right.$ and 0.0006, respectively), which suggested those loci were strongly associated with BC/OC susceptibility. The other tSNPs did not show statistical significance in the multivariate logistic analysis.

Similarly, we analyzed the associations of individual tSNPs in SNAII and TWISTI with BC and OC susceptibility 


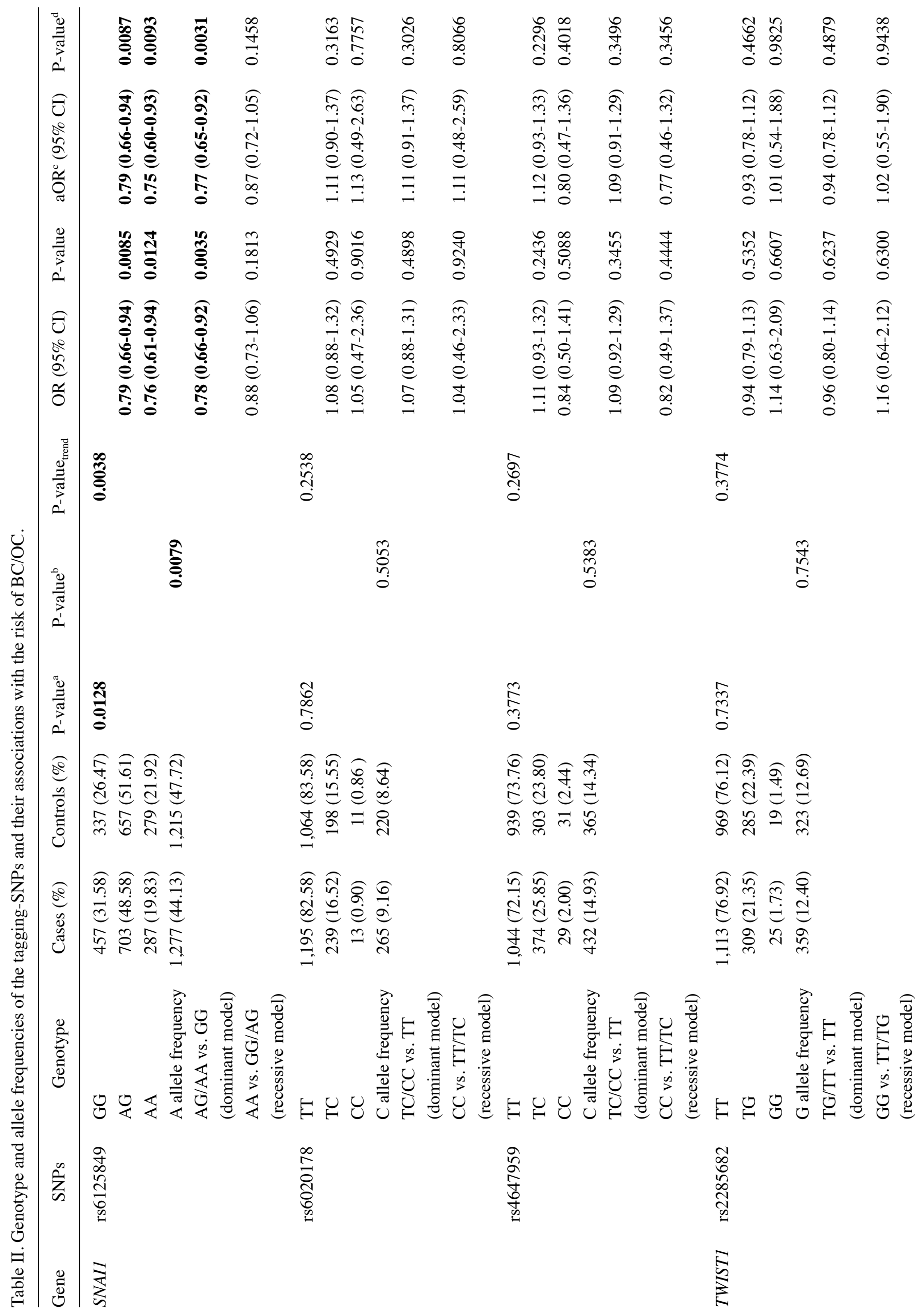




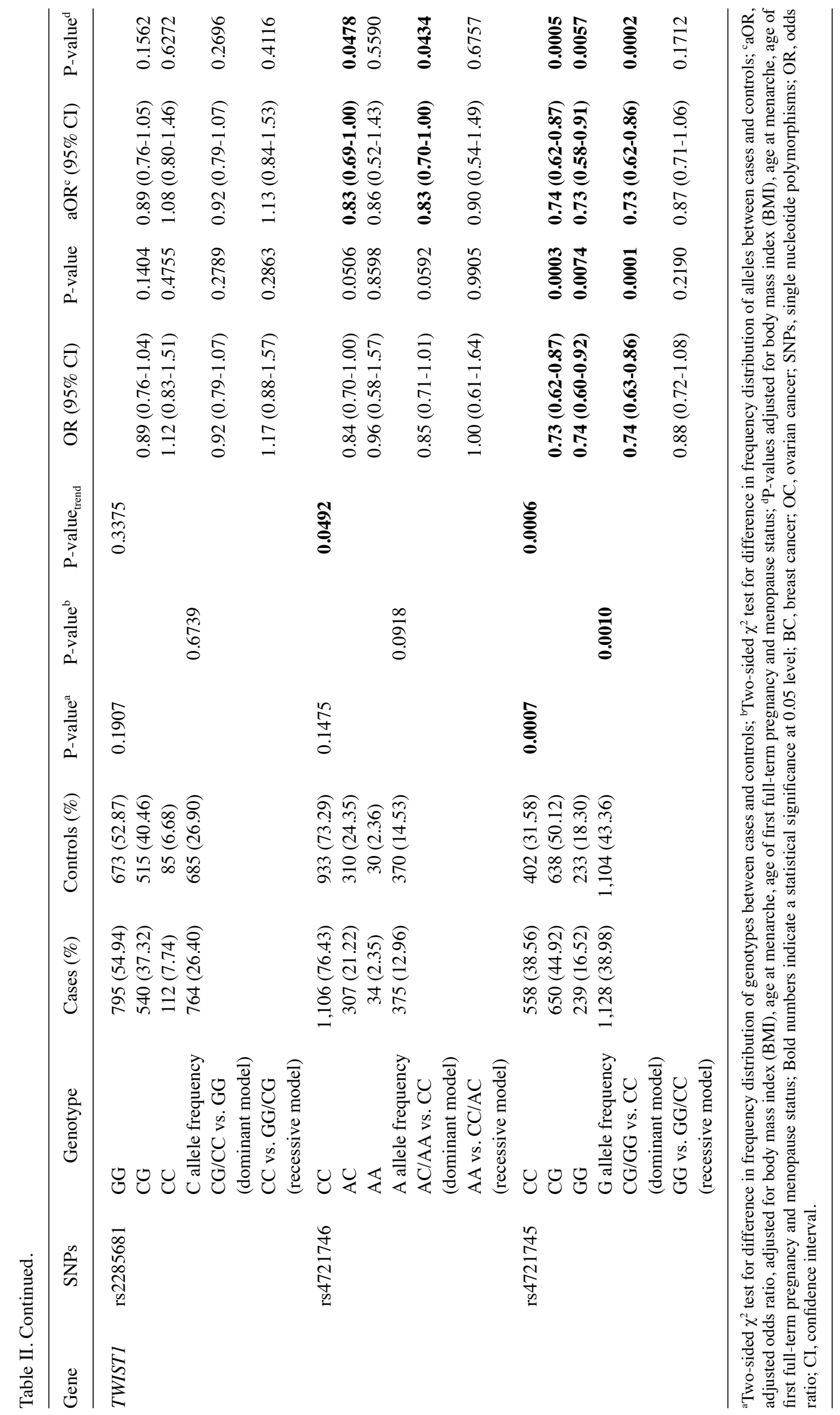


Table III. Multivariate logistic analysis of the BC/OC cases to identify independently related SNPs.

\begin{tabular}{|c|c|c|c|c|c|c|}
\hline Gene & SNPs & Genotype & Cases $(\%)$ & Controls (\%) & $\mathrm{aOR}^{\mathrm{a}}(95 \% \mathrm{CI})$ & P-value ${ }^{b}$ \\
\hline \multirow[t]{5}{*}{ SNAII } & rs6125849 & GG & $457(31.58)$ & 337 (26.47) & & \\
\hline & & $\mathrm{AG}$ & $703(48.58)$ & $657(51.61)$ & $0.80(0.66-0.96)$ & 0.0140 \\
\hline & & $\mathrm{AA}$ & $287(19.83)$ & $279(21.92)$ & $0.77(0.62-0.96)$ & 0.0200 \\
\hline & & $\begin{array}{l}\text { AG/AA vs. GG } \\
\text { (dominant model) }\end{array}$ & & & $0.79(0.66-0.94)$ & 0.0065 \\
\hline & & $\begin{array}{l}\text { AA vs. GG/AG } \\
\text { (recessive model) }\end{array}$ & & & $0.89(0.74-1.07)$ & 0.2183 \\
\hline \multirow[t]{5}{*}{ TWIST1 } & rs4721746 & $\mathrm{CC}$ & $1106(76.43)$ & $933(73.29)$ & & \\
\hline & & $\mathrm{AC}$ & 307 (21.22) & $310(24.35)$ & $0.79(0.65-0.96)$ & 0.0150 \\
\hline & & $\mathrm{AA}$ & $34(2.35)$ & $30(2.36)$ & $0.75(0.44-1.26)$ & 0.2796 \\
\hline & & $\begin{array}{l}\text { AC/AA vs. CC } \\
\text { (dominant model) }\end{array}$ & & & $0.79(0.65-0.95)$ & 0.0107 \\
\hline & & $\begin{array}{l}\text { AA vs. CC/AC } \\
\text { (recessive model) }\end{array}$ & & & $1.04(0.61-1.75)$ & 0.8971 \\
\hline \multirow[t]{5}{*}{ TWIST1 } & rs4721745 & $\mathrm{CC}$ & $558(38.56)$ & $402(31.58)$ & & \\
\hline & & $\mathrm{CG}$ & $650(44.92)$ & $638(50.12)$ & $0.72(0.61-0.86)$ & 0.0002 \\
\hline & & GG & $239(16.52)$ & $233(18.30)$ & $0.73(0.58-0.91)$ & 0.0060 \\
\hline & & $\begin{array}{l}\text { CG/GG vs. CC } \\
\text { (dominant model) }\end{array}$ & & & $0.73(0.62-0.85)$ & 0.0001 \\
\hline & & $\begin{array}{l}\text { GG vs. GG/CC } \\
\text { (recessive model) }\end{array}$ & & & $0.88(0.71-1.07)$ & 0.2004 \\
\hline \multirow[t]{5}{*}{ TWIST1 } & rs 11973396 & AA & $799(55.22)$ & $641(50.35)$ & & \\
\hline & & $\mathrm{AT}$ & $526(36.35)$ & $518(40.69)$ & $0.97(0.79-1.20)$ & 0.7918 \\
\hline & & $\mathrm{TT}$ & $122(8.43)$ & $114(8.96)$ & $1.17(0.79-1.73)$ & 0.4264 \\
\hline & & $\begin{array}{l}\text { AT/TT vs. AA } \\
\text { (dominant model) }\end{array}$ & & & $0.78(0.67-0.92)$ & 0.0024 \\
\hline & & $\begin{array}{l}\text { TT vs. AA/AT } \\
\text { (recessive model) }\end{array}$ & & & $1.21(0.88-1.67)$ & 0.2473 \\
\hline
\end{tabular}

a aOR, adjusted odds ratio, adjusted for age at menarche and age of first full-term pregnancy and other 3 candidate SNPs; ${ }^{\mathrm{P}} \mathrm{P}$-values adjusted for age at menarche and age of first full-term pregnancy and other 3 candidate SNPs; Bold numbers indicate a statistical significance at the 0.05 level; BC, breast cancer; OC, ovarian cancer; SNPs, single nucleotide polymorphisms; OR, odds ratio; CI, confidence interval.

respectively. The three variants (SNAII rs6125849, TWISTI rs4721746, TWIST1 rs4721745) were strongly associated with decreased breast cancer risk $(\mathrm{P}<0.05)$. For ovarian cancer, no $\mathrm{SNP}$ reached statistical significance.

Fine-scale genetic mapping by genotype imputation. Based on the 1000 Genomes dataset, there are 44 variants (MAF> $5 \%$ ) in SNAII and 13 variants (MAF> $5 \%$ ) in TWIST1 in the Chinese Han Beijing population. Using our directly genotyped 7 tSNPs data, 28 variants (22 variants in SNAII and 6 variants in TWIST1) were well-imputed in cases and controls by 'in silico' genotype imputation. Then, we examined the 35 variants for their allelic associations with BC/OC susceptibility using allelic association tests in PLINK software (http://www.cog-genomics.org/plink2/). As shown in Fig. 2, three variants (SNAI1 rs6125849, TWIST1 rs4721745 and TWIST1 rs11973396) demonstrated strong allelic associations with $\mathrm{BC} / \mathrm{OC}$ susceptibility $(\mathrm{OR}=0.87$,
$\mathrm{P}=0.0079 ; \mathrm{OR}=0.83, \mathrm{P}=0.0011 ; \mathrm{OR}=0.87, \mathrm{P}=0.0270$, respectively).

For breast cancer alone, SNAI1 rs6125849 and TWIST1 rs4721745 were associated with decreased $\mathrm{BC}$ risk $(\mathrm{OR}=0.86$, $\mathrm{P}=0.0132 ; \mathrm{OR}=0.83, \mathrm{P}=0.0029)$. For ovarian cancer, however, no SNP reached statistical significance in the gene-wide association study.

Multivariate logistic analysis to identify independently related SNPS. As previously shown in the $\mathrm{BC} / \mathrm{OC}$ case-control study, we identified four protective SNPs by tSNP direct genotyping and genotype imputation, these being SNAI1 rs6125849, TWIST1 rs4721746, TWIST1 rs4721745 and TWIST1 rs11973396. Here, we performed multivariate logistic regression analysis to identify which SNPs are related with BC/OC cancer risk independently. As shown in Table III, all 4 SNPs decreased the BC/OC risk independently. 
A Associations of SNPs in SNAI1 with BC/OC risk

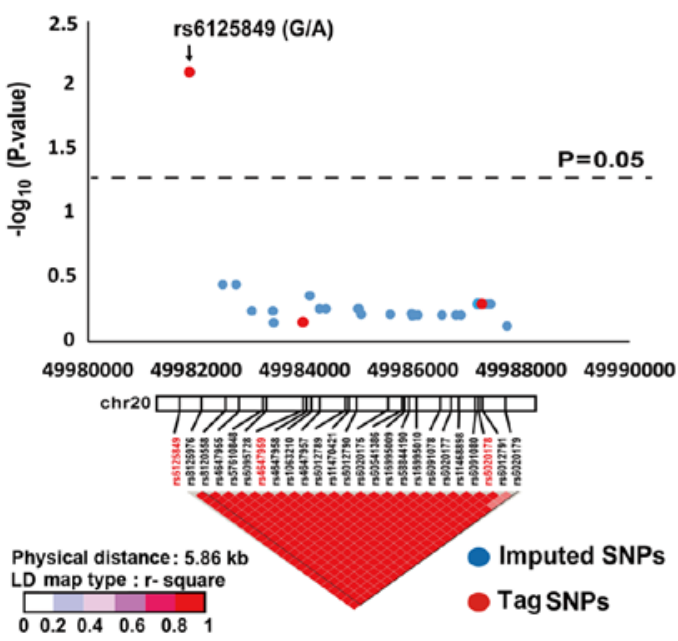

B Associations of SNPs in TWIST1 with BC/OC risk

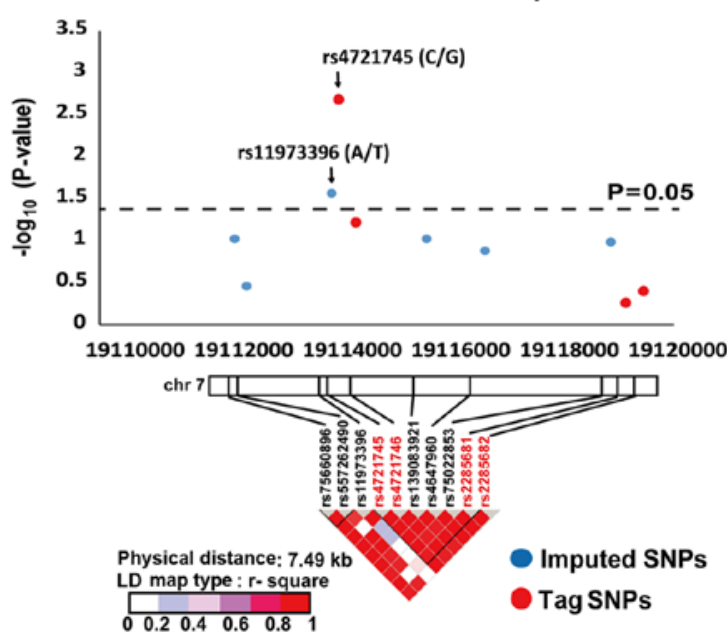

Figure 2. Association of genetic variants in SNAI1 and TWIST1 with risk of BC/OC. Manhattan plots show that rs6125849 in SNAII (A) and rs4721745 and rs11973396 in TWISTI (B) are associated with BC/OC susceptibility. All P-values were calculated using allelic association tests in PLINK software to examine the associations of genetic variants with BC/OC risk. SNPs, single nucleotide polymorphisms; BC, breast cancer; OC, ovarian cancer; LD, linkage disequilibrium.

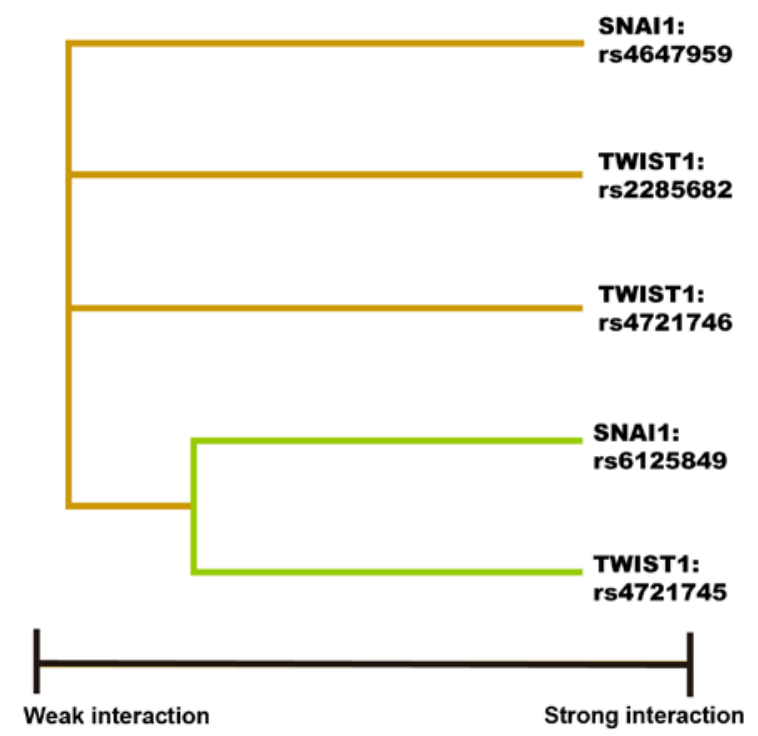

Figure 3. Interaction dendrogram for BC/OC. The loci that strongly interact with each other appear close together at the branches of the tree (green line), whereas the loci with weak interaction appear distant from one another (tawny line). As is shown in the dendrogram, SNAII rs6125849 and TWIST1 rs4721745 display the strongest synergistic interaction. BC, breast cancer; OC, ovarian cancer.

Similarly, multivariate logistic regression was used to analyze whether the three aforementioned protective SNPs for breast cancer (SNAI1 rs6125849, TWIST1 rs4721745 and TWIST1 rs4721746) could affect breast cancer risk independently. It was demonstrated that SNAII rs6125849 and TWIST1 rs4721745 were still strongly associated with breast cancer susceptibility between the $1,161 \mathrm{BC}$ cases and 978 controls, whereas TWIST1 rs4721746 was not further associated with BC susceptibility.

Gene-gene interactions between genetic variants. In the above analyses, the four SNPs, SNAI1 rs6125849, TWIST1 rs4721746, TWIST1 rs4721745 and TWIST1 rs11973396, were demonstrated to be independent genetic variants associated with decreased $\mathrm{BC} / \mathrm{OC}$ risk. Therefore, we performed logistic regression analyses to assess their joint effects on $\mathrm{BC} / \mathrm{OC}$ susceptibility. When all the women carrying none of the 4 protective genotypes at the 4 loci were pooled together to serve as a reference, those harboring three protective genotypes showed significantly lower risk (aOR=0.69, $\mathrm{P}=0.0061$ ), and those harboring 4 protective genotypes demonstrated the lowest risk $(\mathrm{aOR}=0.62, \mathrm{P}=0.0220)$ (Table IV). In subsequent trend test analysis, the dose-dependent effect of the four protective loci was observed with $\mathrm{P}_{\text {trend }}<0.0001$, indicating a synergistic effect of the four loci on $\mathrm{BC} / \mathrm{OC}$ susceptibility.

Similarly, logistic regression demonstrated that SNAII rs6125849 and TWIST1 rs4721745 could jointly affect breast cancer susceptibility as well. Those harboring 2 protective genotypes of SNAII rs6125849 and TWIST1 rs4721745 showed the lowest risk $(\mathrm{aOR}=0.60, \mathrm{P}=0.0011)$. In subsequent trend test analysis, the dose-dependent effect of the two loci was observed with $\mathrm{P}_{\text {trend }}<0.0001$.

Association of high-order interactions with cancer risk by MDR analysis. MDR is a nonparametric and genetic model-free alternative to traditional logistic regression analysis. Since the 7 tSNPs can cover the genetic variants in SNAII and TWISTI gene, we analyzed the interactions among these SNPs by MDR. Table $\mathrm{V}$ shows the best interaction model by MDR analysis for $\mathrm{BC} / \mathrm{OC}$. The best one-factor model for predicting cancer risk was TWIST1 rs4721745 (C>G). It yielded a high testing accuracy of 0.5349 and $\mathrm{CVC}$ of $10 / 10$ with permutation $\mathrm{P}=0.035$, suggesting that this SNP is the primary factor that contributes to $\mathrm{BC} / \mathrm{OC}$ susceptibility compared with the other genetic factors. The best three-factor model for predicting cancer risk included SNAII rs6125849, TWIST1 rs4721745 and TWIST1 rs2285682 (Table V). The interaction dendrogram showed that SNAII rs6125849 and TWIST1 rs4721745 had the strongest synergistic interaction (Fig. 3). 
Table IV. Combination effects of rs6125849, rs4721746, rs4721745 and rs11973396 in the dominant model on BC/OC susceptibility.

\begin{tabular}{|c|c|c|c|c|c|c|c|}
\hline $\begin{array}{l}\text { Number of protective } \\
\text { genotypes }^{\text {a }}\end{array}$ & Cases $(\%)$ & Controls (\%) & $\mathrm{P}_{\text {trend }}$ & OR $(95 \% \mathrm{CI})$ & P-value & $\mathrm{aOR}^{\mathrm{b}}(95 \% \mathrm{CI})$ & P-value ${ }^{c}$ \\
\hline 0 & $162(11.20)$ & $115(9.03)$ & $<0001$ & Reference & & Reference & \\
\hline 1 & $392(27.09)$ & $281(22.07)$ & & $0.99(0.75-1.32)$ & 0.9463 & $1.03(0.78-1.38)$ & 0.8204 \\
\hline 2 & 269 (18.59) & $210(16.50)$ & & $0.91(0.67-1.23)$ & 0.5339 & $0.92(0.68-1.25)$ & 0.6051 \\
\hline 3 & $558(38.56)$ & $590(46.35)$ & & $0.67(0.52-0.88)$ & 0.0033 & $0.69(0.52-0.90)$ & 0.0061 \\
\hline 4 & $66(4.56)$ & 77 (6.05) & & $0.61(0.41-0.91)$ & 0.0166 & $0.62(0.41-0.93)$ & 0.0220 \\
\hline
\end{tabular}

${ }^{a}$ The genetic variants of rs6125849, rs4721746, rs4721745 and rs11973396 were considered as protective genotypes; baOR, adjusted odds ratio, adjusted for body mass index (BMI), age at menarche, age of first full-term pregnancy and menopause status; ${ }^{\circ}$-values adjusted for body mass index (BMI), age at menarche, age of first full-term pregnancy and menopause status; Bold numbers indicate a statistical significance at the 0.05 level; BC, breast cancer; OC, ovarian cancer.

Table V. Association of higher-order gene-gene interactions with BC/OC risk by MDR analysis.

\begin{tabular}{|c|c|c|c|c|}
\hline No. of loci & Best interaction models ${ }^{\mathrm{a}}$ & Testing accuracy & $\mathrm{CVC}$ & $\begin{array}{l}\text { P-value for } \\
\text { permutation } \\
\text { test }\end{array}$ \\
\hline 1 & TWIST1 rs4721745 & 0.5349 & $10 / 10$ & 0.035 \\
\hline 2 & SNAI1 rs6125849+TWIST1 rs4721745 & 0.5340 & $6 / 10$ & 0.042 \\
\hline 3 & $\begin{array}{l}\text { SNAI1 rs6125849+TWIST1 rs2285682+ } \\
\text { TWIST1 rs4721745 }\end{array}$ & 0.5440 & $7 / 10$ & 0.005 \\
\hline 4 & $\begin{array}{l}\text { SNAI1 rs6125849+SNAI1 rs4647959+ } \\
\text { TWIST1 rs4721746+ TWIST1 rs4721745 }\end{array}$ & 0.5350 & $5 / 10$ & 0.035 \\
\hline
\end{tabular}

${ }^{a}$ The model with the maximum testing accuracy and maximum CVC was considered the best model. MDR, multifactor dimensionality reduction; CVC, cross-validation consistency; BC, breast cancer; OC, ovarian cancer.

Table VI. False-positive report probability (FPRP) values for positive results on associations between tagging-SNPs and BC/OC.

\begin{tabular}{|c|c|c|c|c|c|c|c|c|}
\hline \multirow[b]{2}{*}{ Gene/SNP } & \multirow{2}{*}{$\begin{array}{c}\text { Crude OR } \\
(95 \% \mathrm{CI})\end{array}$} & \multirow[b]{2}{*}{ P-value ${ }^{a}$} & \multirow{2}{*}{$\begin{array}{c}\text { Statistical } \\
\text { power }^{b}\end{array}$} & \multicolumn{5}{|c|}{ Prior probability } \\
\hline & & & & 0.25 & 0.1 & 0.01 & 0.001 & 0.0001 \\
\hline \multicolumn{9}{|c|}{ SNAII rs6125849 $(\mathrm{G}>\mathrm{A})$} \\
\hline AG vs. GG & $0.79(0.66-0.94)$ & 0.0085 & 0.9999 & 0.0249 & 0.0711 & 0.4570 & 0.8947 & 0.9884 \\
\hline AA vs. GG & $0.76(0.61-0.94)$ & 0.0124 & 0.9988 & 0.0359 & 0.1005 & 0.5514 & 0.9254 & 0.9920 \\
\hline AG/AA vs. GG & $0.78(0.66-0.92)$ & 0.0035 & 0.8402 & 0.0123 & 0.0361 & 0.2920 & 0.8063 & 0.9766 \\
\hline \multicolumn{9}{|c|}{ TWIST1 rs4721746 (C>A) } \\
\hline AC vs. CC & $0.84(0.70-1.00)$ & 0.0506 & 0.5917 & 0.2042 & 0.4349 & 0.8944 & 0.9884 & 0.9988 \\
\hline AC/AA vs. CC & $0.85(0.71-1.01)$ & 0.0592 & 0.4526 & 0.2818 & 0.5407 & 0.9283 & 0.9924 & 0.9992 \\
\hline \multicolumn{9}{|c|}{ TWIST1 rs4721745 (C>G) } \\
\hline CG vs. CC & $0.73(0.62-0.87)$ & 0.0003 & 0.9890 & 0.0009 & 0.0027 & 0.0292 & 0.2326 & 0.7521 \\
\hline GG vs. CC & $0.74(0.60-0.92)$ & 0.0074 & 0.9829 & 0.0221 & 0.0635 & 0.4270 & 0.8826 & 0.9869 \\
\hline CG/GG vs. CC & $0.74(0.63-0.86)$ & 0.0001 & 0.9483 & 0.0003 & 0.0009 & 0.0103 & 0.0953 & 0.5132 \\
\hline
\end{tabular}

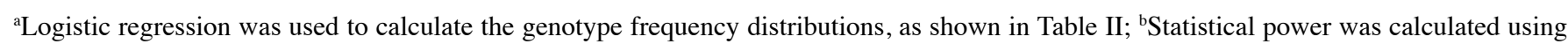
the number of observations in the subgroup and the OR and P-values in this table; Bold numbers indicate a noteworthy finding with an FPRP value <0.2; SNPs, single nucleotide polymorphisms; BC, breast cancer; OC, ovarian cancer; OR, odds ratio. 


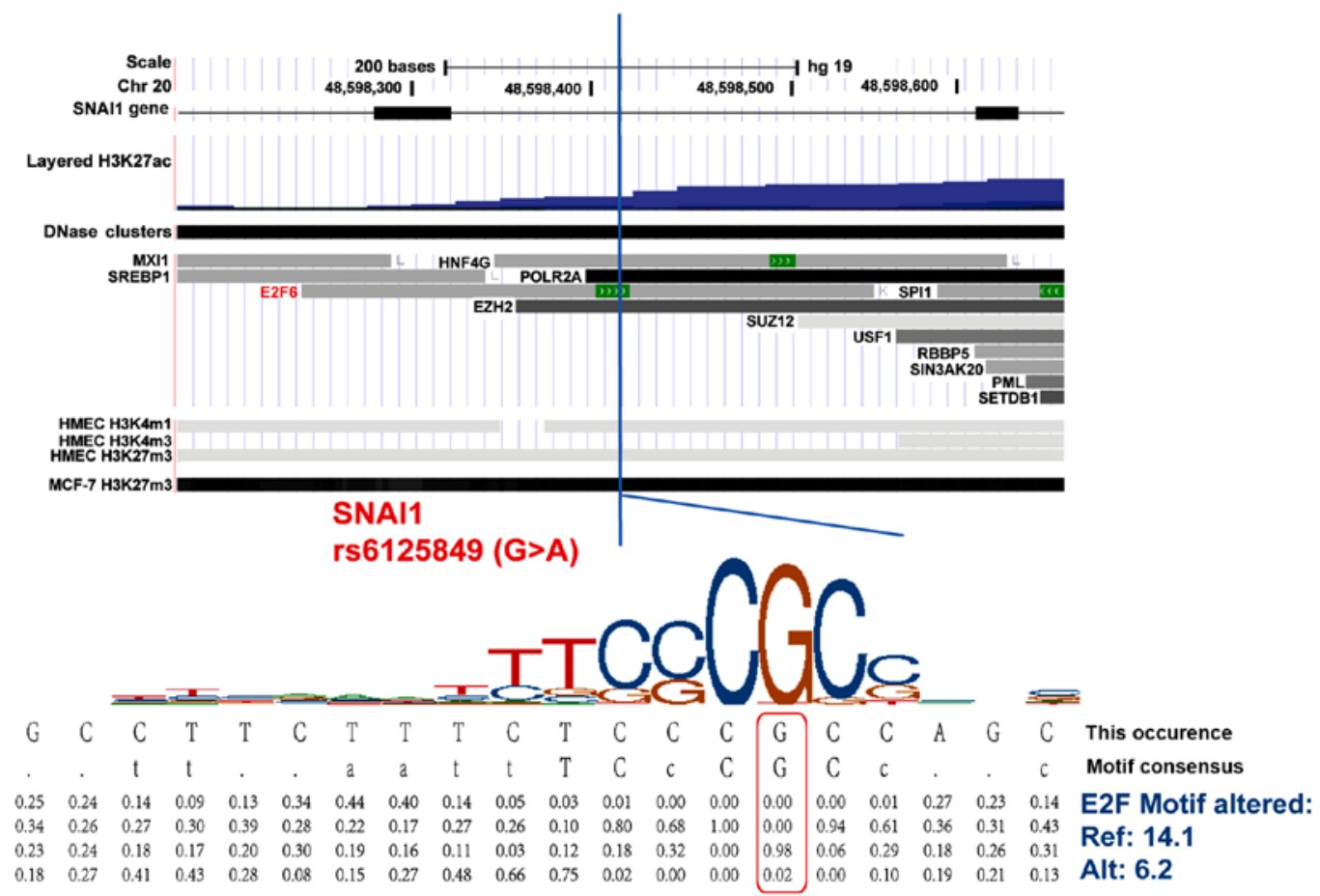

Figure 4. A comprehensive functional annotation of BC/OC susceptibility locus SNAII rs6125849. The vertical blue line indicates the location of SNAII rs6125849. According to HaploReg and RegulomeDB, SNAII rs6125849 is located in the DNase I hypersensitive site and the histone modification region in the human mammary epithelial cell line HMEC and breast cancer cell line MCF-7. In addition, this locus binds to many transcription factors, including E2F6 (upper panel). Moreover, the minor allele A of SNAIl rs6125849 (G>A) may decrease its binding affinity with E2F family proteins (lower panel). BC, breast cancer; OC, ovarian cancer.

Similarly, MDR analysis was performed in BC and OC separately, and the interaction between SNAII rs6125849 and TWIST1 rs4721745 remained in both breast cancer and ovarian cancer (data not shown).

False-positive report probability and statistical power analysis. All the above analyses indicated that SNAII rs6125849, TWIST1 rs4721745 and rs4721746 were strongly associated with $\mathrm{BC} / \mathrm{OC}$ susceptibility. To decide whether these findings deserve attention or are 'noteworthy', we calculated false-positive report probability (FPRP) and statistical power. As shown in Table VI, at the FPRP threshold of 0.2 and prior probability level of 0.1, the associations of SNAI1 rs6125849 and TWISTI rs4721745 with BC/OC susceptibility remained noteworthy.

Functional annotation. Functional annotations were conducted for these 'noteworthy' SNPs SNAII rs6125849 and TWIST1 rs4721745 using HaploReg (http://www.broadinstitute.org/mammals/haploreg/haploreg.php) and RegulomeDB (http://regulome.stanford.edu/). In ovarian tissues, breast cancer cell line MCF-7, and human mammary epithelial cell line HMEC, SNAII rs6125849 (G>A) is located in the region with histone methylation, acetylation modifications and many transcriptional factor binding sites, indicating a role of expressional regulation in this region (Fig. 3). In addition, the minor allele A of SNAIl rs6125849 (G>A) alters its binding affinity with transcription factor E2F6 based on the HaploReg database (Fig. 4). The TWIST1 rs4721745 is located in the region with histone modifications in human mammary epithelial cell line HMEC. Using Jaspar database, it predicted that the minor allele $\mathrm{G}$ of TWIST1 rs4721745 (C>G) altered its binding affinity with transcription factor TCF11-MafG. Overall, SNAII rs6125849 and TWIST1 rs4721746 may be functional loci.

\section{Discussion}

To the best of our knowledge, this is the first gene-wide SNP study to comprehensively evaluate the association of genetic variants in SNAII and TWISTI with the risk of breast cancer (BC) and ovarian cancer (OC) in Chinese Han women.

In this study, we applied multiple strategies including tagging-SNP genotyping, genotype imputation, logistic regression (LR), MDR and functional annotation to systematically evaluate the association of $\mathrm{BC} / \mathrm{OC}$ susceptibility with germline variants in SNAII and TWISTI among Chinese Han women. By LR, we discovered that three tSNPs (SNAII rs6125849, TWIST1 rs4721746, and TWIST1 rs4721745) were associated with decreased risks of BC/OC. Since a fine-mapping study with high-density SNPs within the target region may be helpful in identifying the causal variants, we used genotype imputation to analyze more variants among the 2 genes, and revealed an imputed SNP TWIST1 rs11973396 to be associated with decreased $\mathrm{BC} / \mathrm{OC}$ risk as well. Moreover, we demonstrated the joint effects of these protective loci by LR and MDR analyses, and found that women carrying minor alleles of both SNAII rs6125849 and TWIST1 rs4721745 could decrease their risk of $\mathrm{BC} / \mathrm{OC}$ by $40 \%$.

According to our single-locus analysis, TWIST1 rs4721745 $(\mathrm{C}>\mathrm{G})$, located in the 3 ' flanking region of the gene, might be the strongest protective locus against $\mathrm{BC} / \mathrm{OC}$ in our population. A recently published study on endometrial cancer susceptibility from our laboratory also showed rs 4721745 to 
be a protective locus in the $\mathrm{CHB}$ population, which is consistent with our results (35). Using web-based software F-SNP (http://compbio.cs.queensu.ca/F-SNP/) (36), this polymorphism is predicted to have transcriptional regulation function by TFSearch and Consite tools (FS score 0.208). In addition, by using miRNA prediction module PicTar and TargetScan from UCSC genome browser, miR-33/381 is predicted to be the twist-specific target regulatory elements (37). Zhou et al found that the miR-33a level was negatively correlated with the Twist level in Saos-2 cells, and inhibition of miR-33a increased cisplatin-induced cell apoptosis, which was reversed by knockdown of Twist (38). Our functional annotation also indicated that rs4721745 was located in a region with histone methylation modifications such as $\mathrm{H} 3 \mathrm{~K} 4 \mathrm{me} 3$, a modification usually associated with active transcription of nearby genes, suggesting that the variation of this locus may alter TWISTI expression by influencing its transcription level. Jaspar database predicted that the minor allele G of TWIST1 rs4721745 $(\mathrm{C}>\mathrm{G})$ altered its binding affinity with transcription factor TCF11-MafG. Based on these findings, we predict that this protective locus may be involved in TWIST1 transcription and alter its biological functions.

SNAII inhibits the expression of epithelial markers and activates mesenchymal molecules, thus participating in tumor epithelial-mesenchymal transition and metastasis (39). Our results showed that SNAII rs6125849 (located near 5' flanking region) decreased $\mathrm{BC}$ risk. The 3DSNP (http://cbportal. org/3dsnp/) predicted that this locus was located in the loop between the promoter and enhancer with histone modifications and many transcriptional factor binding sites. HaploReg database predicted that the minor allele A of SNAII rs6125849 $(\mathrm{G}>\mathrm{A})$ altered its binding affinity with transcription factor E2F6. We presumed this protective locus might affect SNAIl transcription, thereby inhibiting tumor development. Regarding SNAII rs4647958 (Val118Ala), also known as c.353T $>C$, Lei et al genotyped this missense variation in 2,072 lung cancer cases and 2,077 control subjects, and demonstrated a protective effect of rs4647958 on lung cancer susceptibility $(\mathrm{OR}=0.76,95 \% \mathrm{CI}=0.65-0.90, \mathrm{P}=0.018)(40)$. In this study, however, we did not find any association between rs4647958 and BC/OC risk. It is possible that rs4647958 plays a different role in lung cancer and $\mathrm{BC} / \mathrm{OC}$.

Our study inevitably has limitations. First, we only analyzed the association of common genetic variants (MAF $\geq 0.05$ ) with BC/OC susceptibility, but did not analyze the association of low frequency or very low frequency of genetic variants (MAF <0.05), which might have led to the omission of important variants. Also, due to the small sample size of the subgroups, various results may be chance findings, although we used various statistical methods to control the false positives.

In summary, this study suggests that genetic variants in SNAI1 and TWIST1 are associated with BC/OC susceptibility. Our data also suggest a synergistic effect of those related loci on $\mathrm{BC} / \mathrm{OC}$ risk. Nevertheless, further validation studies are needed for further determination of the truly causal SNPs and their exact functional mechanisms. These cancer-associated SNPs have the potential to improve our future ability of personalized evaluation of $\mathrm{BC} / \mathrm{OC}$ susceptibility and facilitate the identification of high-risk subgroups in the general population.

\section{Acknowledgements}

Not applicable.

\section{Funding}

The present study was supported by the National Natural Science Foundation of China (nos. 81672790 and 81621063) and the Open Project of the Key Laboratory of Genomic and Precision Medicine, Chinese Academy of Sciences.

\section{Availability of data and materials}

The datasets used during the present study are available from the corresponding author upon reasonable request.

\section{Authors' contributions}

YJW, XXT and WGF conceived and designed the study. YJW, LYZ and LY performed the experiments. YJW, ZFW, RS, LY and YHG analyzed the data. FM, YTX, LC, DGL, XHL and YLS designed the study and contributed with reagents/materials/analysis tools. YJW, ZFW, YHG and RS wrote the paper. XXT, WGF and YHG revised the paper. All authors read and approved the manuscript and agree to be accountable for all aspects of the research in ensuring that the accuracy or integrity of any part of the work are appropriately investigated and resolved.

\section{Ethics approval and consent to participate}

The present study was approved by the Peking University IRB (reference no. IRB00001052-11029). We obtained written informed consents from all BC cases and control women. Since we could not obtain the contact information of ovarian cancer patients treated before 2011, PKU IRB approved our application to waive informed consent for the archived samples collected before April 2011. This study only used these samples. We used all the data/samples anonymously.

\section{Patient consent for publication}

Not applicable.

\section{Competing interests}

The authors declare that they have no competing interests.

\section{References}

1. Chen W, Zheng R, Zhang S, Zhao P, Zeng H and Zou X: Report of cancer incidence and mortality in China, 2010. Ann Transl Med 2: 61-85, 2014.

2. Jordan SJ, Cushing-Haugen KL, Wicklund KG, Doherty JA and Rossing MA: Breast-feeding and risk of epithelial ovarian cancer. Cancer Causes Control 23: 919-927, 2012.

3. Zheng L, Song A, Ruan Y, Chen L, Liu D, Li X, Guo H, Han J, Li Y, Tian X, et al: Genetic polymorphisms in AURKA, BRCA1, $C C N E 1$ and $C D K 2$ are associated with ovarian cancer susceptibility among Chinese Han women. Cancer Epidemiol 37: 639-646, 2013.

4. Steiner E, Klubert D and Knutson D: Assessing breast cancer risk in women. Am Fam Physician 78: 1361-1366, 2008. 
5. Bodelon C, Wentzensen N, Schonfeld SJ, Visvanathan K, Hartge P, Park Y and Pfeiffer RM: Hormonal risk factors and invasive epithelial ovarian cancer risk by parity. Br J Cancer 109: 769-776, 2013

6. Scollen S, Luccarini C, Baynes C, Driver K, Humphreys MK, Garcia-Closas M, Figueroa J, Lissowska J, Pharoah PD, Easton DF, et al: TGF- $\beta$ signaling pathway and breast cancer susceptibility. Cancer Epidemiol Biomarkers Prev 20: 1112-1119, 2011.

7. Antoniou A, Pharoah PD, Narod S, Risch HA, Eyfjord JE, Hopper JL, Loman N, Olsson H, Johannsson O, Borg A, et al: Average risks of breast and ovarian cancer associated with $B R C A 1$ or $B R C A 2$ mutations detected in case Series unselected for family history: A combined analysis of 22 studies. Am J Hum Genet 72: 1117-1130, 2003.

8. Antoniou AC and Easton DF: Models of genetic susceptibility to breast cancer. Oncogene 25: 5898-5905, 2006.

9. Yoshida $\mathrm{K}$ and Miki Y: Role of BRCA1 and BRCA2 as regulators of DNA repair, transcription, and cell cycle in response to DNA damage. Cancer Sci 95: 866-871, 2004.

10. Rahdar M, Inoue $\mathrm{T}$, Meyer $\mathrm{T}$, Zhang $\mathrm{J}$, Vazquez $\mathrm{F}$ and Devreotes PN: A phosphorylation-dependent intramolecular interaction regulates the membrane association and activity of the tumor suppressor PTEN. Proc Natl Acad Sci USA 106 : 480-485, 2009.

11. Vousden KH: p53: Death star. Cell 103: 691-694, 2000.

12. Hollestelle A, Wasielewski M, Martens JW and Schutte M: Discovering moderate-risk breast cancer susceptibility genes. Curr Opin Genet Dev 20: 268-276, 2010.

13. Schork NJ, Murray SS, Frazer KA and Topol EJ: Common vs. rare allele hypotheses for complex diseases. Curr Opin Genet Dev 19: 212-219, 2009.

14. Liu CT, Lin H and Lin H: Functional analysis of HapMap SNPs. Gene 511: 358-363, 2012.

15. Stram DO, Haiman CA, Hirschhorn JN, Altshuler D, Kolonel LN, Henderson BE and Pike MC: Choosing haplotype-tagging SNPS based on unphased genotype data using a preliminary sample of unrelated subjects with an example from the Multiethnic Cohort Study. Hum Hered 55 27-36, 2003.

16. Li Y, Willer C, Sanna S and Abecasis G: Genotype imputation. Annu Rev Genomics Hum Genet 10: 387-406, 2009.

17. Nothnagel M, Ellinghaus D, Schreiber S, Krawczak M and Franke A: A comprehensive evaluation of SNP genotype imputation. Hum Genet 125: 163-171, 2009.

18. Clark AG: The role of haplotypes in candidate gene studies. Genet Epidemiol 27: 321-333, 2004.

19. Wang Y, Shi J, Chai K, Ying X and Zhou BP: The role of snail in EMT and tumorigenesis. Curr Cancer Drug Targets 13: 963-972, 2013.

20. Jin H, Yu Y, Zhang T, Zhou X, Zhou J, Jia L, Wu Y, Zhou BP and Feng Y: Snail is critical for tumor growth and metastasis of ovarian carcinoma. Int J Cancer 126: 2102-2111, 2010.

21. Moody SE, Perez D, Pan TC, Sarkisian CJ, Portocarrero CP, Sterner CJ, Notorfrancesco KL, Cardiff RD and Chodosh LA: The transcriptional repressor Snail promotes mammary tumor recurrence. Cancer Cell 8: 197-209, 2005.

22. Jiang X, Guo D, Li W, Yu T, Zhou J and Gong J: Combination Twist1 and CA15-3 in axillary lymph nodes for breast cancer prognosis. Mol Med Rep 15: 1123-1134, 2017.

23. Terauchi M, Kajiyama H, Yamashita M, Kato M, Tsukamoto $H$, Umezu T, Hosono S, Yamamoto E, Shibata K, Ino K, et al: Possible involvement of TWIST in enhanced peritoneal metastasis of epithelial ovarian carcinoma. Clin Exp Metastasis 24: 329-339, 2007.
24. Hosono S, Kajiyama H, Terauchi M, Shibata K, Ino K, Nawa A and Kikkawa F: Expression of Twist increases the risk for recurrence and for poor survival in epithelial ovarian carcinoma patients. Br J Cancer 96: 314-320, 2007.

25. Matsusaka S, Zhang W, Cao S, Hanna DL, Sunakawa Y, Sebio A, Ueno M, Yang D, Ning Y, Parekh A, et al: TWIST1 polymorphisms predict survival in patients with metastatic colorectal cancer receiving first-line bevacizumab plus oxaliplatin-based chemotherapy. Mol Cancer Ther 15: 1405-1411, 2016.

26. Grimberg J, Nawoschik S, Belluscio L, McKee R, Turck A and Eisenberg A: A simple and efficient non-organic procedure for the isolation of genomic DNA from blood. Nucleic Acids Res 17: 8390, 1989.

27. Pikor LA, Enfield KSS, Cameron H and Lam WL: DNA extraction from paraffin embedded material for genetic and epigenetic analyses. J Vis Exp 49: e2763, 2011.

28. Barrett JC, Fry B, Maller J and Daly MJ: Haploview: Analysis and visualization of LD and haplotype maps. Bioinformatics 21: 263-265, 2005

29. Lewis CM: Genetic association studies: Design, analysis and interpretation. Brief Bioinform 3: 146-153, 2002.

30. He J, Wang F, Zhu J, Zhang R, Yang T, Zou Y and Xia H: Association of potentially functional variants in the $X P G$ gene with neuroblastoma risk in a Chinese population. J Cell Mol Med 20: 1481-1490, 2016

31. Wacholder S, Chanock S, Garcia-Closas M, El Ghormli L and Rothman N: Assessing the probability that a positive report is false: An approach for molecular epidemiology studies. J Natl Cancer Inst 96: 434-442, 2004

32. Browning BL and Browning SR: A unified approach to genotype imputation and haplotype-phase inference for large data sets of trios and unrelated individuals. Am J Hum Genet 84: 210-223, 2009.

33. Liu L, Wu C, Wang Y, Zhong R, Wang F, Zhang X, Duan S, Lou J, Yu D, Tan W, et al: Association of candidate genetic variations with gastric cardia adenocarcinoma in Chinese population: A multiple interaction analysis. Carcinogenesis 32: 336-342, 2011.

34. Ghosh J, Pradhan S and Mittal B: Multilocus analysis of hormonal, neurotransmitter, inflammatory pathways and genome-wide associated variants in migraine susceptibility. Eur J Neurol 21: 1011-1020, 2014.

35. Yang L, Wang YJ, Zheng LY, Jia YM, Chen YL, Chen L, Liu DG, Li XH, Guo HY, Sun YL, et al: Genetic polymorphisms of TGFB1, TGFBRI, SNAII and TWISTl are associated with endometrial cancer susceptibility in chinese Han women. PLoS One 11: e0155270,2016

36. Lee PH and Shatkay H: F-SNP: Computationally predicted functional SNPs for disease association studies. Nucleic Acids Res 36: D820-D824, 2008.

37. Landgraf P, Rusu M, Sheridan R, Sewer A, Iovino N, Aravin A, Pfeffer S, Rice A, Kamphorst AO, Landthaler M, et al: A mammalian microRNA expression atlas based on small RNA library sequencing. Cell 129: 1401-1414, 2007.

38. Zhou Y, Huang Z, Wu S, Zang X, Liu M and Shi J: miR-33a is up-regulated in chemoresistant osteosarcoma and promotes osteosarcoma cell resistance to cisplatin by down-regulating TWIST. J Exp Clin Cancer Res 33: 12, 2014.

39. Dhasarathy A, Phadke D, Mav D, Shah RR and Wade PA: The transcription factors Snail and Slug activate the transforming growth factor-beta signaling pathway in breast cancer. PLoS One 6: e26514, 2011

40. Yang L, Yang X, Ji W, Deng J, Qiu F, Yang R, Fang W, Zhang L, Huang D, Xie C, et al: Effects of a functional variant c.353T $>C$ in snail on risk of two contextual diseases. Chronic obstructive pulmonary disease and lung cancer. Am J Respir Crit Care Med 189: 139-148, 2014 